\title{
Uma implementação de modelos matemáticos de máquinas síncronas em MATLAB/SIMULINK como atividade interdisciplinar
}

\author{
Alcedir Luis Finkler*, Maurício de Campos ${ }^{\star *}$, Paulo Sérgio Sausen ${ }^{\star \star *}$, \\ Airam Teresa Zago Romcy Sausen ${ }^{\star \star \star \star}$
}

\section{Resumo}

Na disciplina de máquinas elétricas, ofertada em cursos técnicos e cursos de engenharia voltados à área elétrica, estuda-se um dos principais componentes utilizados em sistemas de geração de energia elétrica: a máquina síncrona. A compreensão do funcionamento dessa máquina requer um conhecimento prévio de disciplinas como física e matemática. Física para o estudo do momento de inércia, coeficiente de atrito, interação entre campos magnéticos, e matemática para a compreensão das equações utilizadas na construção dos modelos. Logo, este tema, além de ser muito importante para compreensão dos sistemas elétricos, envolve a aplicação de conhecimentos adquiridos em diversas disciplinas dos respectivos cursos. Para a realização das aulas, algumas atividades podem ser desenvolvidas em laboratório, porém, há alguns parâmetros nessas máquinas que são inacessíveis por meio de experimentação prática e precisam ser analisados via simulação computacional. Entre os possíveis softwares para realização dessas simulações está o MATLAB/SIMULINK. Em uma proposta realizada com alunos do curso Técnico Subsequente em Eletromecânica desenvolveu-se o presente trabalho, que reúne os principais modelos matemáticos de máquinas síncronas e descreve a sua implementação em MATLAB/SIMULINK. A atividade proposta permitiu aos alunos uma melhor compreensão do princípio de funcionamento dessas máquinas.

Palavras-Chave: Ângulo de carga; Dinâmica de sistemas de potência; Modelos de máquinas síncronas; Modelos de sistema de potência; Monitoramento de sistemas de potência.

* Mestre em Modelagem Matemática e Computacional pela Universidade Regional do Noroeste do Estado do Rio Grande do Sul, Brasil. Docente EBTT do Instituto Federal Farroupilha, Brasil. E-mail: alcedir.finkler@iffarroupilha. edu.br

* Doutor em Engenharia Elétrica pela Universidade Federal de Campina Grande, Brasil. Professor Adjunto I da Universidade Regional do Noroeste do Estado do Rio Grande do Sul, Brasil. E-mail: campos@unijui.edu.br

*** Doutor em Engenharia Elétrica pela Universidade Federal de Campina Grande, Brasil. Professor Adjunto III da Universidade Regional do Noroeste do Estado do Rio Grande do Sul, Brasil. E-mail: sausen@unijui.edu.br

*** Doutora em Engenharia Elétrica pela Universidade Federal de Campina Grande, Brasil. Professor Adjunto III da Universidade Regional do Noroeste do Estado do Rio Grande do Sul, Brasil. E-mail: airam@unijui.edu.br 


\section{Introdução}

Modelos matemáticos de máquinas síncronas têm sido amplamente estudados nas últimas décadas. $\mathrm{O}$ conhecimento desses modelos é de fundamental importância para o desenvolvimento de estudos voltados ao sistema elétrico de potência (SEP). Desse modo, Ghahremani (2011) descreve o ângulo de carga de máquinas síncronas como um dos parâmetros mais importantes para monitoração e controle de estabilidade no SEP.

Na construção dos modelos de máquinas síncronas é considerado um sistema de coordenadas com origem no centro do rotor, conforme ilustrado na Fig. 1.a. Esse sistema possui um dos eixos alinhado ao bobinado de campo, chamado de eixo direto (eixo-d), e o segundo eixo, deslocado em 90 graus elétricos, que chama-se de eixo em quadratura (eixo-q). Sob os eixos direto e em quadratura são consideradas bobinas fictícias para representar as correntes parasitas e correntes que circulam nos bobinados amortecedores. Logo, como essas bobinas são fictícias nas disciplinas de máquinas elétricas, a visualização do comportamento dos fluxos magnéticos e das correntes precisa ser realizada por meio de simulação computacional.

Diferentes modelos de máquinas síncronas podem ser propostos de acordo com o número de bobinas que será considerado no rotor da máquina. Os modelos são classificados como Modelo i.j, onde i corresponde ao número de bobinas considerado sob o eixo-d, e j corresponde ao número de bobinas considerado sob o eixo-q. Krishna (2014) introduz a modelagem de máquinas síncronas e apresenta, inicialmente, um modelo (Modelo 2.2) que considera duas bobinas sob o eixo-d (F e 1D) e duas bobinas sob o eixo-q (1Q e 2Q), conforme ilustrado na Fig. 1.a. Em seguida, descreve as principais simplificações apresentadas na literatura para esse modelo.

Contudo, diferentes modelos de máquinas síncronas são utilizados para obtenção de parâmetros, como o ângulo de carga. Como exemplo, é possível citar o trabalho de Ghahremani (2011), que descreve um método para estimar o ângulo de carga direcionado ao estudo de estabilidade após a ocorrência de falhas na rede. $\mathrm{O}$ autor descreve que o efeito das bobinas amortecedoras pode ser negligenciado quando a dinâmica das máquinas em períodos subtrasitórios não for de interesse. No desenvolvimento, devido a mudanças abruptas nos valores de impedância da máquina, explica que o ângulo de carga obtido possui um valor incorreto durante a falha (GHAHREMANI, 2011). O trabalho de Venkatasubramanian (2004), por sua vez, descreve a importância de 
estudos direcionados a estimar os estados de máquinas síncronas. Assim, apresenta um método utilizando o Modelo 1.0 para obtenção do ângulo de carga. Justifica sua escolha ao salientar que esse modelo é de fácil representação. Já Jha e Chakrabarti (2015) descrevem um método que utiliza unidades de medição fasorial e o Modelo 2.1 para obtenção do ângulo de carga. Caberia avaliar quão diferentes são os modelos na obtenção do ângulo de carga de máquinas síncronas.

Destarte, neste trabalho é descrita a metodologia de simulação dos principais modelos de máquinas síncronas com o objetivo de obter o ângulo de carga a partir da utilização do SIMULINK. Os resultados obtidos para os ângulos de carga são comparados ao modelo de máquinas síncronas disponível na biblioteca do SIMULINK, que utiliza os parâmetros padrões com valores em percentuais (p.u.). Esses resultados foram comparados e para isso foram utilizados os coeficientes de correlação de Pearson. As principais contribuições deste trabalho são: permitir que os alunos do curso Técnico Subsequente em Eletromecânica tenham uma melhor visualização da implementação das equações, que tenham uma melhor compreensão dos ganhos e perdas obtidos com a simplificação dos modelos matemáticos, e orientar na escolha do modelo de máquinas síncronas mais adequado a ser utilizado em estudos relacionados ao ângulo de carga de máquinas conectadas ao SEP em função do objetivo (conhecer valores em regime transitório ou conhecer valores em regime permanente) e da máquina a ser estudada (máquinas de polos lisos ou máquinas de polos salientes).

A metodologia utilizada nesta produção, para a simulação de máquinas síncronas e obtenção do ângulo de carga com os diferentes modelos, é descrita na Seção 2 . Na Seção 3 são discutidos os resultados obtidos. Na Seção 4 é realizada a conclusão deste trabalho.

Figura 1: Bobinas máquina síncrona: (a) plano abc, (b) plano dq0.

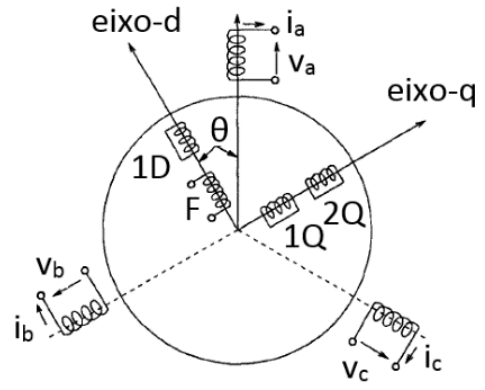

(a)

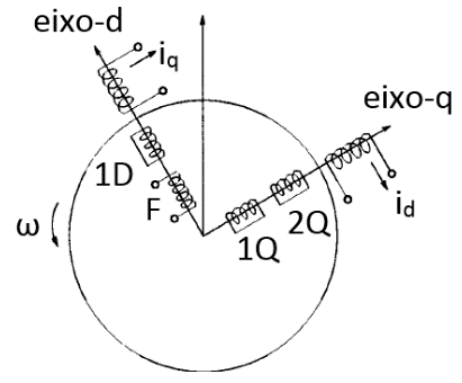

(b) 


\section{Simulação de máquinas síncronas}

O SIMULINK possui, em sua biblioteca, um bloco que permite a simulação de máquinas síncronas ao inserir os parâmetros padrões da máquina com seus valores em p.u. Esse modelo está ilustrado na Fig. 2. O SIMULINK permite, por meio do módulo PowerSim, realizar a inicialização de máquinas obtendo os parâmetros de tensão a ser aplicada ao bobinado de campo (Vf1) e potência mecânica a ser aplicada ao eixo (Pm), com objetivo de obter determinados valores de potência ativa e reativa. Para realização das simulações, considerou-se uma máquina síncrona de 30 $\mathrm{kVA}, 4$ polos, operando em regime permanente com $10 \%$ da potência ativa e fator de potência unitário (FP) no período de simulação de 0 a 10 segundos. Decorrido esse período, a potência mecânica aplicada ao eixo é elevada de forma a obter $100 \%$ da potência ativa e mantendo $\mathrm{FP}=1$.

Figura 2: Diagrama de referência.

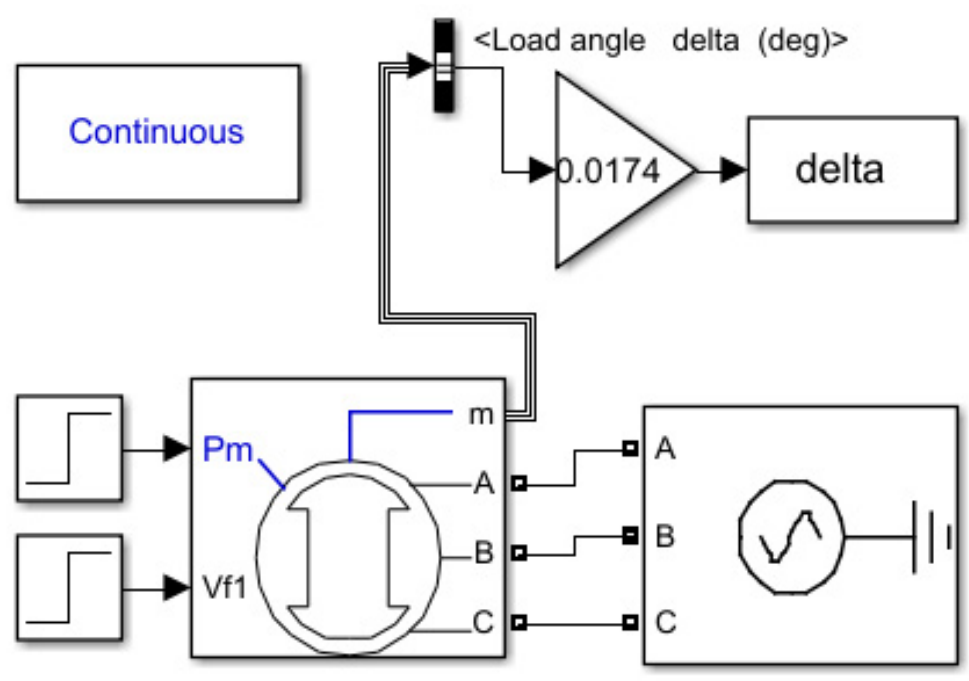

Como neste trabalho o objetivo é comparar o ângulo de carga obtido com os diferentes modelos, utilizou-se como entrada em Pm um sinal degrau. Em aplicações práticas, devido a inércia dos equipamentos, é impossível a implementação de um degrau a Pm, porém, em simulação, com objetivo de comparar diferentes modelos matemáticos, este ensaio permite uma melhor visualização da dinâmica do modelo à tal entrada quando comparada a entradas em rampa. Boldea (2016), no capítulo 
5.12.1, apresenta um estudo para análise do comportamento de máquinas síncronas a um degrau aplicado ao torque mecânico.

O diagrama utilizado para simulação, ilustrado na Fig. 2, consiste em uma máquina síncrona conectada diretamente a um barramento infinito (representando o SEP). O ângulo de carga apresenta o valor em graus. Utilizou-se um ganho $\mathrm{k}=$ (๘/180) para converter o valor em radianos. No desenvolvimento das equações serão utilizados os seguintes parâmetros:

$\mathrm{R}_{\mathrm{a}}$ Resistência de armadura;

$\mathrm{R}_{\mathrm{L}}$ Resistência da carga;

$\mathrm{X}_{\mathrm{d}}, \mathrm{X}_{\mathrm{d}}, \mathrm{X}_{\mathrm{d}}$ ” (no SIMULINK Xd, Xdd, Xddd) Reatância síncrona, transitória e subtransitória de eixo direto;

$\mathrm{X}_{q}, \mathrm{X}_{\mathrm{q}}, \mathrm{X}_{\mathrm{q}}$ ” (no SIMULINK Xq, Xqd, Xqdd) Reatância síncrona, transitória e subtransitória do eixo em quadratura;

Td', Td" (no SIMULINK Tdd, Tddd) Constante de tempo transitória e subtransitória de curto circuito do eixo direto;

Tq', Tq" (no SIMULINK Tqd, Tqdd) Constante de tempo transitória e subtransitória de curto circuito do eixo em quadratura;

$\mathrm{T}_{\mathrm{d} 0}, \mathrm{~T}_{\mathrm{d} 0}$ " Constante de tempo transitória e subtransitória de circuito aberto do eixo direto;

$\mathrm{T}_{\mathrm{q} 0}, \mathrm{~T}_{\mathrm{q} 0}$ ” Constante de tempo transitória e subtransitória de circuito aberto do eixo em quadratura;

$\boldsymbol{\delta}$ (no SIMULINK delta) Ângulo de carga;

$\omega$ Velocidade angular do eixo da máquina;

$\omega_{0}$ Velocidade angular do campo magnético girante do estator;

$\omega_{\mathrm{B}}$ Velocidade angular nominal da máquina;

$i_{d}, i_{q}$ Corrente no eixo direto e eixo em quadratura;

$\mathrm{v}_{\mathrm{d}}, \mathrm{v}_{\mathrm{q}}$ Tensão no eixo direto e eixo em quadratura;

$\psi_{\mathrm{d}}, \psi_{\mathrm{q}}$ (no SIMULINK Psid, Psiq) Fluxo magnético concatenado nas bobinas do eixo direto e eixo em quadratura do estator;

$\psi_{\mathrm{F}}, \psi_{1 \mathrm{D}}$ (no SIMULINK PsiF, Psi1D) Fluxo magnético concatenado no bobinado de campo e fluxo magnético concatenado no bobinado fictício considerado sob eixo direto; 
$\Psi_{1 Q}, \Psi_{2 Q}$ (no SIMULINK Psi1Q, Psi2Q) Fluxo magnético concatenado nos bobinados fictícios 1 e 2 considerados sob eixo em quadratura;

$\mathrm{H}$ Constante de inércia;

$\mathrm{T}_{\mathrm{m}}$ Torque mecânico;

V Tensão de linha da rede.

Os valores para esses parâmetros utilizados nas simulações estão listados no Anexo A. Nas seções seguintes estão descritos os procedimentos para simulação dos diferentes modelos apresentados por Krishna (2014).

\section{Modelo da máquina síncrona em p.u. (Modelo 2.2)}

O Modelo 2.2 apresentado por Krishna (2014), considera, no rotor, duas bobinas posicionadas sob o eixo direto e duas bobinas sob o eixo em quadratura, conforme ilustrado na Fig. 1b.

As equações das máquinas síncronas quando escritas em função das correntes, tensões e fluxos magnéticos das bobinas de armadura, A, B e C, apresentam não linearidades. Para contornar essas não linearidades, essas bobinas são substituídas por duas bobinas fictícias, localizadas sob o eixo-d e eixo-q, que se movem alinhadas às bobinas do rotor, conforme ilustrado na Fig. 1.b. Essa substituição é feita por meio das transformadas de Park, também conhecidas como transformadas dq0 (KUNDUR, 1994).

As equações utilizadas para determinar os estados da máquina síncrona, escritas em função dos parâmetros padrões em valores por unidade (p.u.), são apresentadas de Eq (1) à Eq (10) (KRISHNA, 2014).

$$
\begin{aligned}
& \frac{d \bar{\psi}_{d}}{d_{t}}=-\omega \bar{\psi}_{q}-\omega_{B} \bar{R}_{a} \bar{i}_{d}-\omega \overline{\mathrm{v}}_{d} \\
& \frac{d \bar{\psi}_{q}}{d_{t}}=-\omega \bar{\psi}_{d}-\omega_{B} \bar{R}_{a} \bar{i}_{q}-\omega \overline{\mathrm{v}}_{q}
\end{aligned}
$$




$$
\begin{aligned}
& \frac{d \bar{\psi}_{F}}{d_{t}}=\frac{1}{T_{d}{ }^{\prime}}\left(\left(-\bar{\psi}_{F}+\bar{\psi}_{d}+\left(\frac{\bar{X}_{d}^{\prime}}{\bar{X}_{d}-\bar{X}_{d}^{\prime}}\right)\right) E_{f}\right) \\
& \frac{d \bar{\psi}_{1 D}}{d_{t}}=\frac{1}{T_{d}^{\prime \prime}}\left(-\bar{\psi}_{1 D}+\bar{\psi}_{d}\right) \\
& \frac{d \bar{\psi}_{1 Q}}{d_{t}}=\frac{1}{T_{q}^{\prime}}\left(-\bar{\psi}_{1 Q}+\bar{\psi}_{q}\right) \\
& \frac{d \bar{\psi}_{2 Q}}{d_{t}}=\frac{1}{T_{q} "}\left(-\bar{\psi}_{2 Q}+\bar{\psi}_{q}\right) \\
& \frac{d \delta}{d_{t}}=\omega-\omega_{0} \\
& \frac{d \omega}{d_{t}}=\frac{\omega_{B}}{2 H}\left(T_{m}-\bar{\psi}_{d} \bar{i}_{q}+\bar{\psi}_{q} \bar{i}_{d}\right) \\
& \bar{i}_{d}=\frac{1}{\bar{X}_{d}{ }^{\prime \prime}} \bar{\psi}_{d}+\left(\frac{1}{\bar{X}_{d}}-\frac{1}{\bar{X}_{d}^{\prime}}\right) \bar{\psi}_{F}+\left(\frac{1}{\bar{X}_{d}^{\prime}}-\frac{1}{\bar{X}_{d}{ }^{\prime \prime}}\right) \bar{\psi}_{1 D} \\
& \bar{i}_{q}=\frac{1}{\bar{X}_{q}{ }^{\prime \prime}} \bar{\psi}_{q}+\left(\frac{1}{\bar{X}_{q}}-\frac{1}{\bar{X}_{q}^{\prime}}\right) \bar{\psi}_{1 Q}+\left(\frac{1}{\bar{X}_{q}^{\prime}}-\frac{1}{\bar{X}_{q}{ }^{\prime \prime}}\right) \bar{\psi}_{2 Q}
\end{aligned}
$$

As tensões nos eixos diretos e em quadratura são obtidas por Eq (11) e Eq (12).

$$
\begin{aligned}
& \bar{v}_{d}=-\overline{\mathrm{V}} \operatorname{sen}(\delta) \\
& \bar{v}_{q}=\overline{\mathrm{V}} \cos (\delta)
\end{aligned}
$$


A implementação das Eq (11) e Eq (12) no SIMULINK, para obtenção das tensões nos eixos direto e em quadratura, é ilustrada na Fig. 3.

Figura 3: Tensões $v_{d}$ e $v_{q}$, Modelo 2.2.

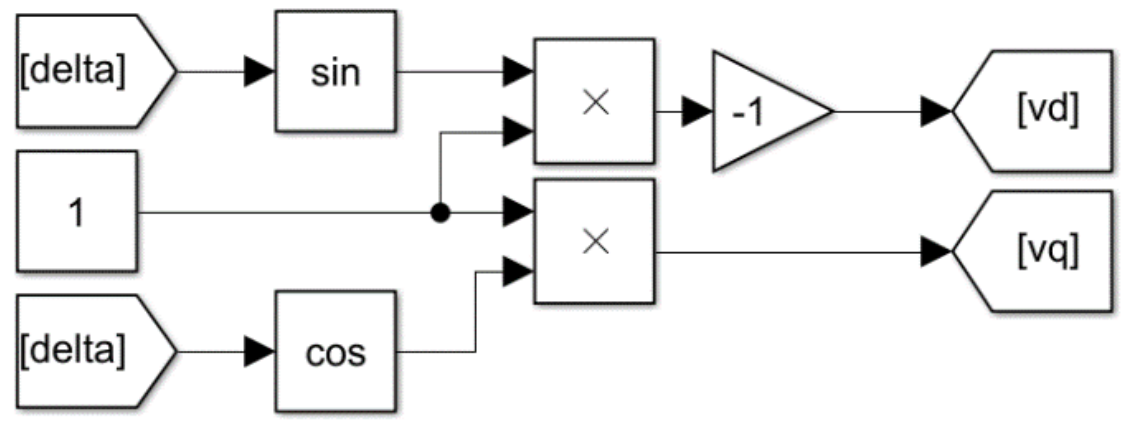

A implementação das equações mecânicas Eq (7) e Eq (8) no SIMULINK é ilustrada na Fig. 4.

Figura 4: Equações mecânicas, Modelo 2.2.

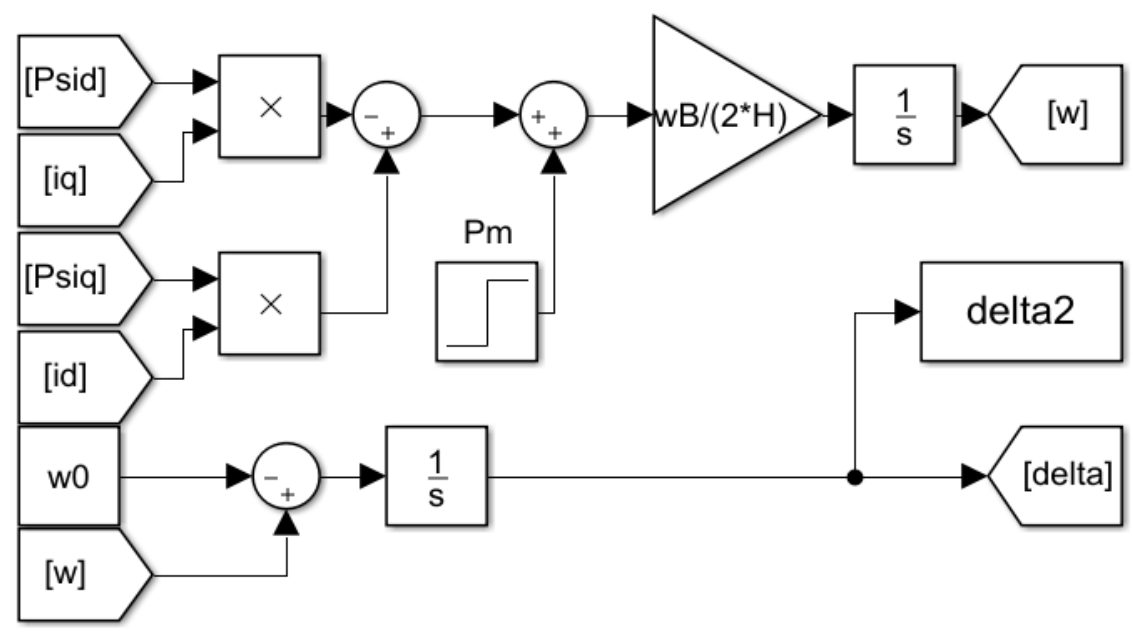

A implementação, no SIMULINK, das equações dos fluxos magnéticos relacionadas às bobinas $\psi_{\mathrm{F}}$ e $\psi_{1 \mathrm{D}}$ do eixo direto do rotor Eq (3) e Eq (4) é ilustrada na Fig. 5. 
Figura 5: Equações dos fluxos no eixo direto, Modelo 2.2.

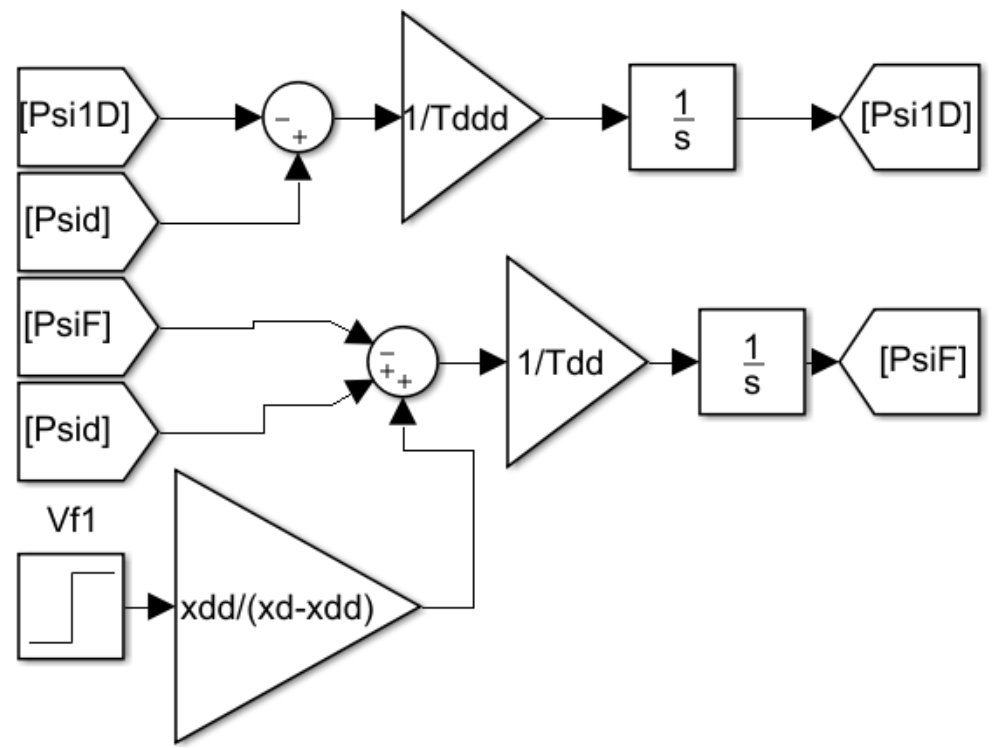

Aimplementação, no SIMULINK, das equações dos fluxos magnéticos relacionadas às bobinas $\Psi_{1 \mathrm{Q}}$ e $\Psi_{2 \mathrm{Q}}$ do eixo em quadratura do rotor Eq (5) e Eq (6) é ilustrada na Fig. 6.

Figura 6: Equações dos fluxos no eixo em quadratura, Modelo 2.2.

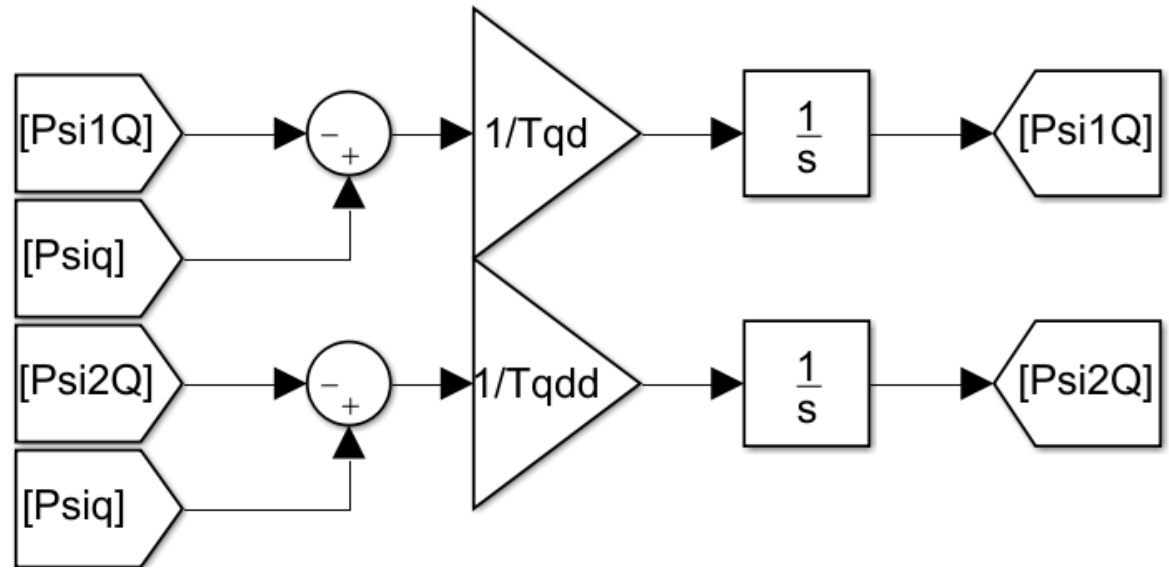

Para cálculo do ângulo de carga, os transientes do estator podem ser negligenciados considerando $\mathrm{d} \psi_{\mathrm{d}} / \mathrm{dt}=0, \mathrm{~d} \psi_{\mathrm{q}} / \mathrm{dt}=0, \omega=\omega_{\mathrm{B}}$. Logo, Eq (1) e Eq (2) são escritas como Eq (13) e Eq (14) (KUNDUR, 1994; KRISHNA, 2014). 


$$
\begin{aligned}
& 0=-\bar{\psi}_{q}-\bar{R}_{a} \bar{i}_{d}-\overline{\mathrm{v}}_{d} \\
& 0=-\bar{\psi}_{d}-\bar{R}_{a} \bar{i}_{q}-\overline{\mathrm{v}}_{q}
\end{aligned}
$$

A implementação das equações relacionadas às bobinas do estator $\mathrm{Eq}$ (13) e Eq (14) no SIMULINK é ilustrada na Fig. 7.

Figura 7: Equações dos fluxos no estator, Modelo 2.2.

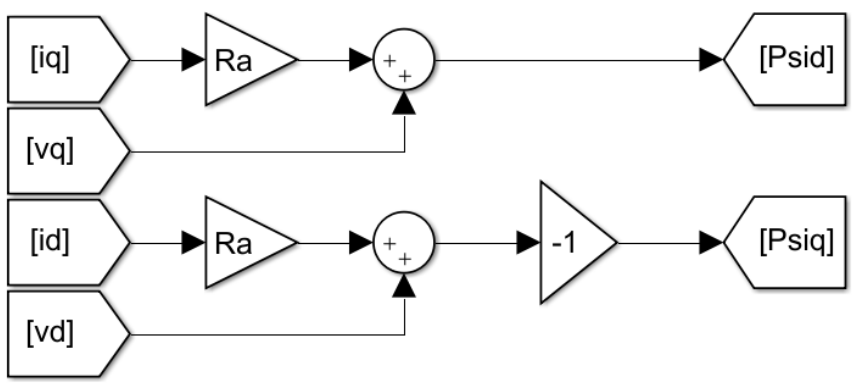

As equações para obtenção das correntes nos eixos direto e em quadratura, descritas em Eq (9) e Eq (10), são ilustradas na Fig. 8.

Figura 8: Equações das correntes, Modelo 2.2.

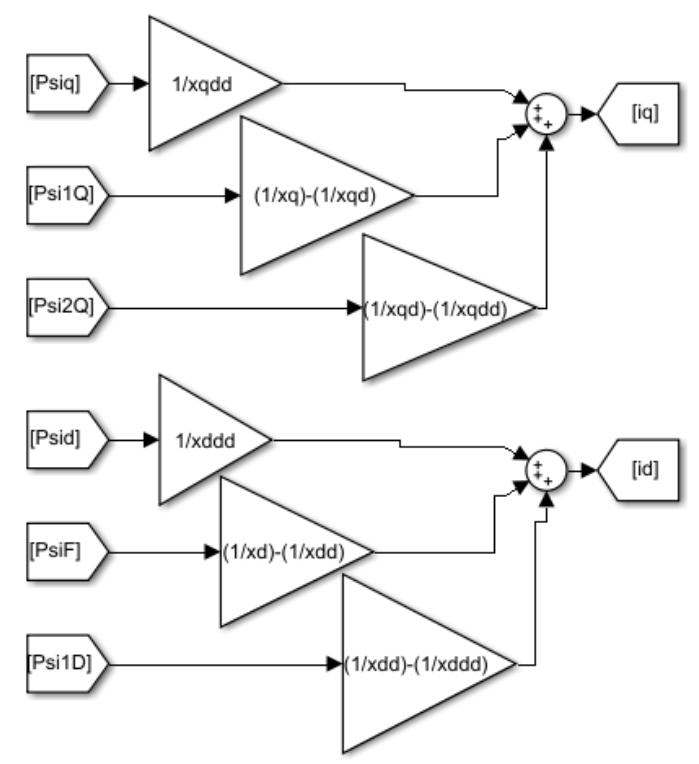


Para realização da simulação, atribui-se os valores dos degraus Vf1 e Pm iguais aos valores ajustados no modelo de referência. Em seguida, é possível comparar o ângulo de carga obtido com os diferentes modelos, como ilustrado na Fig. 9.

Figura 9: Ângulo de carga com Modelo de referência e Modelo 2.2.

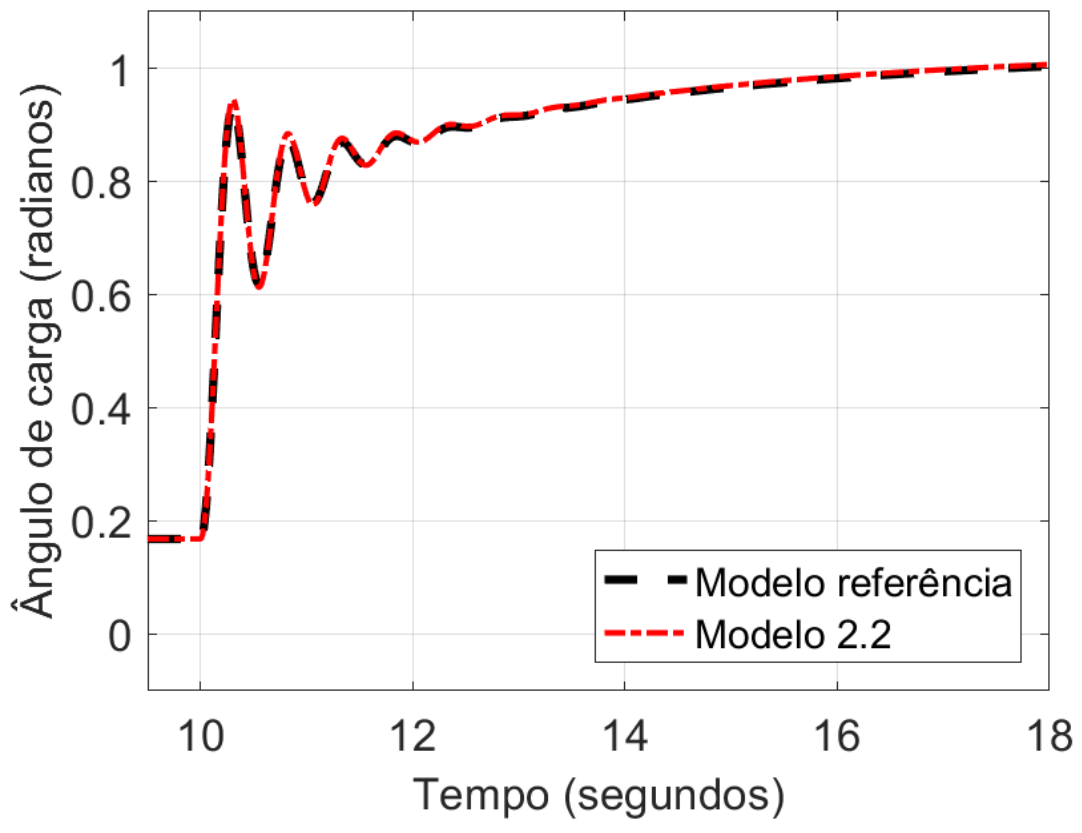

\section{Modelo 2.1}

Uma simplificação no Modelo 2.2 pode ser obtida ao reduzir uma bobina no eixo em quadratura do rotor fazendo $\mathrm{T}_{\mathrm{q}^{\mathrm{g}}}=0, \bar{\Psi}_{2 Q}=\bar{\Psi}_{q}$, logo:

$$
\begin{aligned}
& \frac{d \bar{\psi}_{F}}{d_{t}}=\frac{1}{T_{d}{ }^{\prime}}\left(\left(-\bar{\psi}_{F}+\bar{\psi}_{d}+\left(\frac{\bar{X}_{d}^{\prime}}{\bar{X}_{d}-\bar{X}_{d}^{\prime}}\right)\right) E_{f}\right) \\
& \frac{d \bar{\psi}_{1 D}}{d_{t}}=\frac{1}{T_{d}{ }^{\prime \prime}}\left(-\bar{\psi}_{1 D}+\bar{\psi}_{d}\right)
\end{aligned}
$$




$$
\begin{aligned}
& \frac{d \bar{\psi}_{1 Q}}{d_{t}}=\frac{1}{T_{q}{ }^{\prime}}\left(-\bar{\psi}_{1 Q}+\bar{\psi}_{q}\right) \\
& \frac{d \delta}{d_{t}}=\omega-\omega_{0} \\
& \frac{d \omega}{d_{t}}=\frac{\omega_{B}}{2 H}\left(T_{m}-\bar{\psi}_{d} \bar{i}_{q}+\bar{\psi}_{q} \bar{i}_{d}\right) \\
& \bar{i}_{d}=\frac{1}{\bar{X}_{d}{ }^{\prime \prime}} \bar{\psi}_{d}+\left(\frac{1}{\bar{X}_{d}}-\frac{1}{\bar{X}_{d}^{\prime}}\right) \bar{\psi}_{F}+\left(\frac{1}{\bar{X}_{d}^{\prime}}-\frac{1}{\bar{X}_{d}{ }^{\prime \prime}}\right) \bar{\psi}_{1 D} \\
& \bar{i}_{q}=\frac{1}{\bar{X}_{q}{ }^{\prime \prime}} \bar{\psi}_{q}+\left(\frac{1}{\bar{X}_{q}}-\frac{1}{\bar{X}_{q}^{\prime}}\right) \bar{\psi}_{1 Q}
\end{aligned}
$$

Para simulação do Modelo 2.1, o diagrama para obtenção dos fluxos magnéticos no eixo em quadratura passa a ser conforme ilustrado na Fig. 10.

Figura 10: Equações dos fluxos no eixo em quadratura no Modelo 2.1.

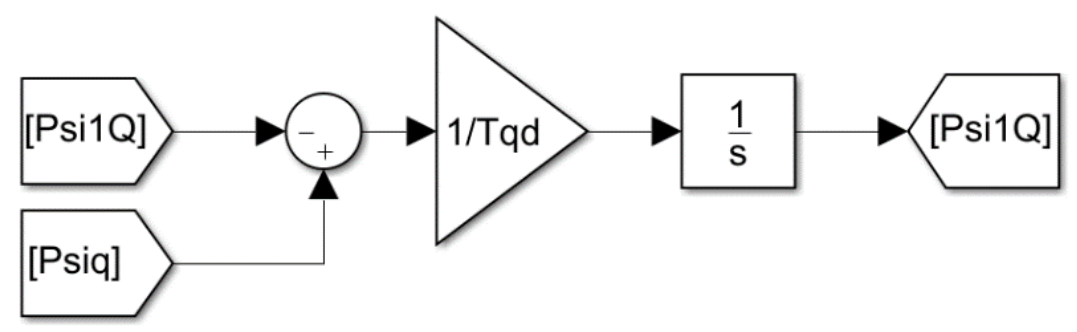

O diagrama das correntes, no Modelo 2.1, passa a ser conforme ilustrado na Fig. 11. 
Figura 11: Equações das correntes no Modelo 2.1.

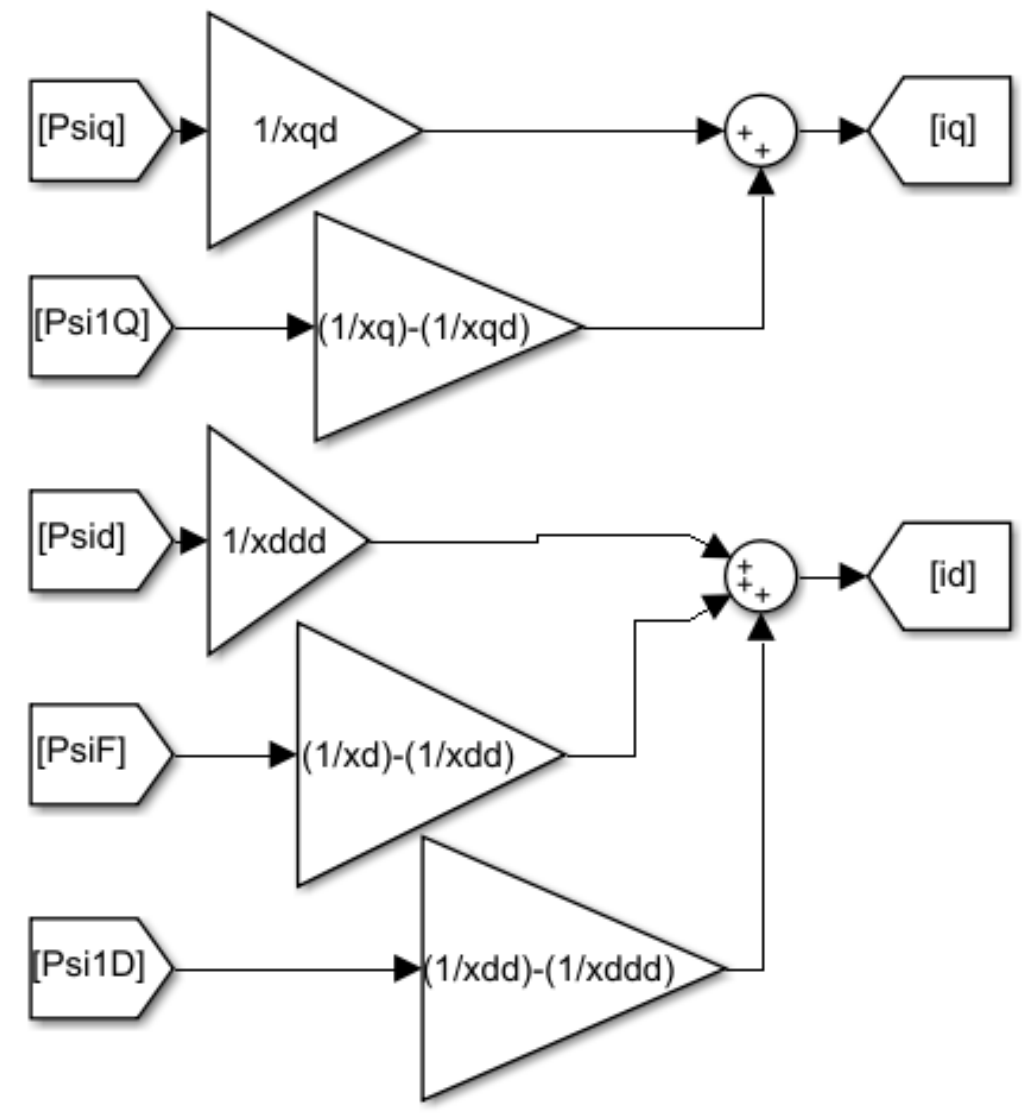

Os fluxos magnéticos no estator permanecem conforme Fig. 7, o fluxo magnético no eixo direto permanece conforme Fig. 5, tensões $\mathrm{v}_{\mathrm{d}}$ e $\mathrm{v}_{\mathrm{q}}$ ficam conforme Fig. 3, e as equações mecânicas continuam conforme Fig. 4.

A Fig. 12 ilustra a comparação entre o ângulo de carga obtido com o Modelo de referência e o Modelo 2.1. 
Figura 12: Ângulo de carga com Modelo de referência e Modelo 2.1.

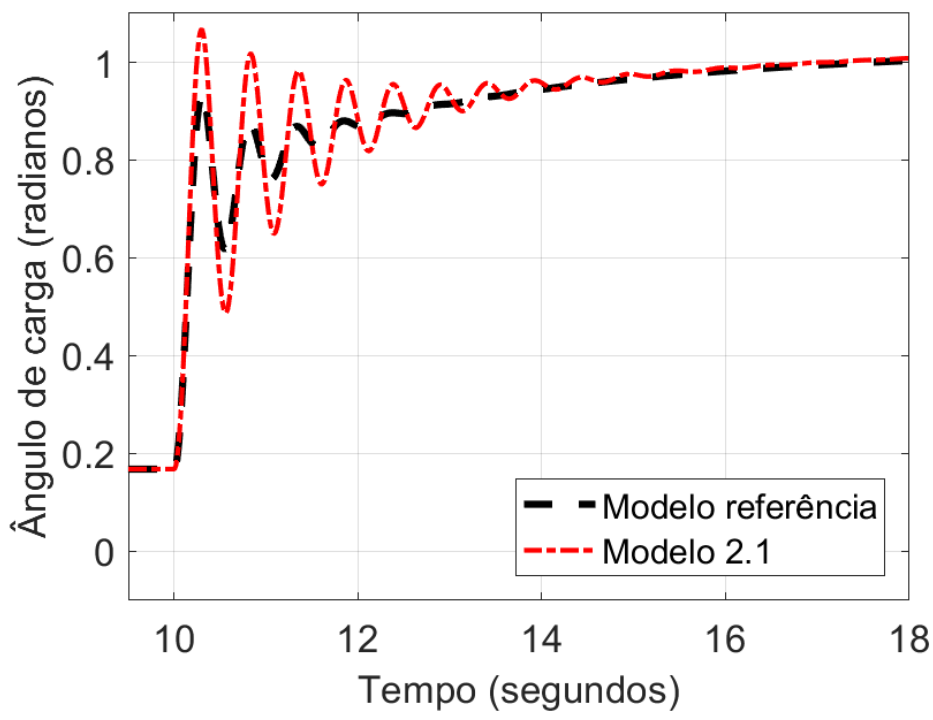

\section{Modelo 1.1}

Uma simplificação no Modelo 2.1 pode ser obtida reduzindo uma bobina no eixo direto do rotor fazendo $\mathrm{T}_{\mathrm{d} "}=0, \bar{\psi}_{1 D}=\bar{\psi}_{d}$, logo:

$$
\begin{aligned}
& \frac{d \bar{\psi}_{F}}{d_{t}}=\frac{1}{T_{d}{ }^{\prime}}\left(\left(-\bar{\psi}_{F}+\bar{\psi}_{d}+\left(\frac{\bar{X}_{d}^{\prime}}{\bar{X}_{d}-\bar{X}_{d}^{\prime}}\right)\right) E_{f}\right) \\
& \frac{d \bar{\psi}_{1 Q}}{d_{t}}=\frac{1}{T_{q}{ }^{\prime}}\left(-\bar{\psi}_{1 Q}+\bar{\psi}_{q}\right) \\
& \frac{d \delta}{d t}=\omega-\omega_{0}
\end{aligned}
$$




$$
\begin{aligned}
& \frac{d \omega}{d_{t}}=\frac{\omega_{B}}{2 H}\left(T_{m}-\bar{\psi}_{d} \bar{i}_{q}+\bar{\psi}_{q} \bar{i}_{d}\right) \\
& \bar{i}_{d}=\frac{1}{\bar{X}_{d}{ }^{\prime}} \bar{\psi}_{d}+\left(\frac{1}{\bar{X}_{d}}-\frac{1}{\bar{X}_{d}^{\prime}}\right) \bar{\psi}_{F} \\
& \bar{i}_{q}=\frac{1}{\bar{X}_{q}^{\prime \prime}} \bar{\psi}_{q}+\left(\frac{1}{\bar{X}_{q}}-\frac{1}{\bar{X}_{q}^{\prime}}\right) \bar{\psi}_{1 Q}
\end{aligned}
$$

Para simulação do Modelo 1.1, o diagrama para obtenção dos fluxos magnéticos no eixo direto e no eixo em quadratura passa a ser conforme ilustrado na Fig. 13.

Figura 13: Equações dos fluxos no rotor do Modelo 1.1.

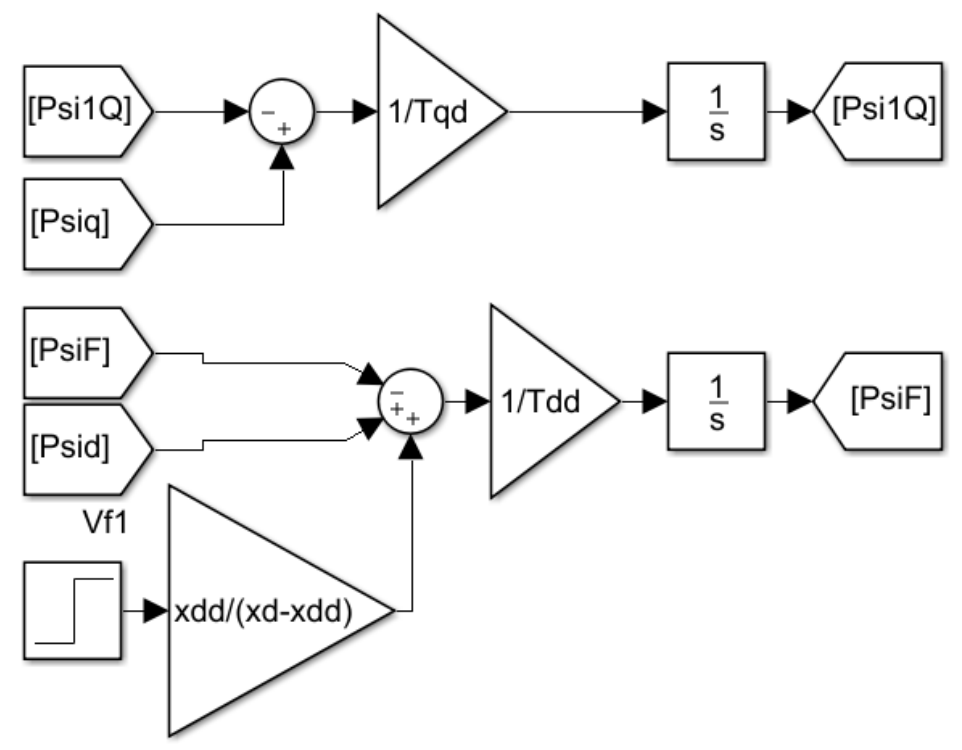

O diagrama das correntes no eixo direto e eixo em quadratura no Modelo 1.1 passa a ser como ilustrado na Fig. 14. Os fluxos magnéticos no estator permanecem conforme Fig. 7, tensões $\mathrm{v}_{\mathrm{d}}$ e $\mathrm{v}_{\mathrm{q}}$ ficam conforme Fig. 3 e as equações mecânicas continuam conforme Fig. 4. 
Figura 14: Equações das correntes no Modelo 1.1.

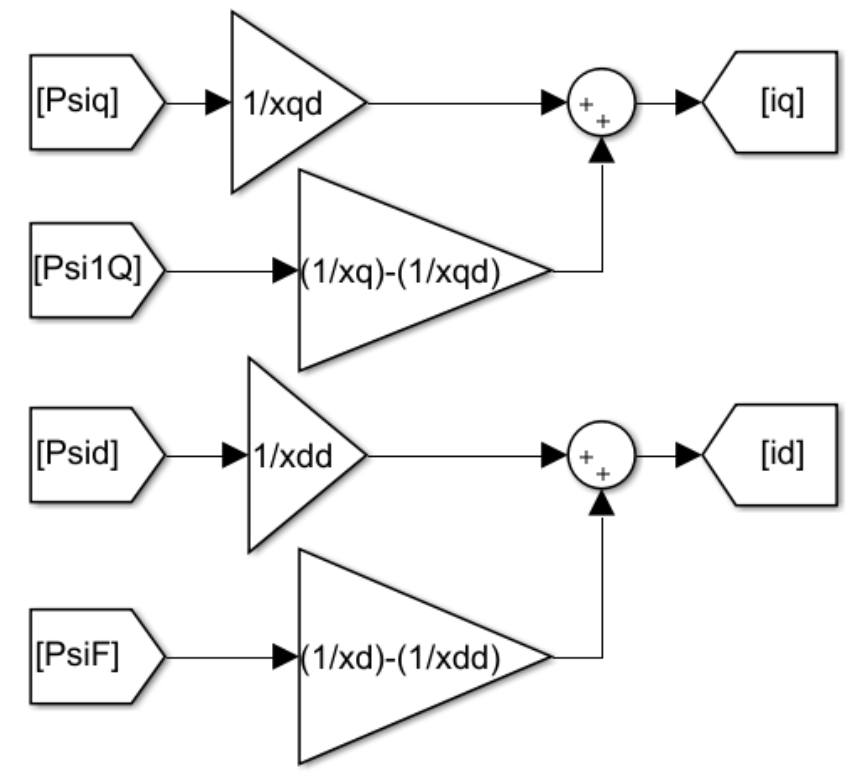

A Fig. 15 ilustra a comparação entre o ângulo de carga obtido com o Modelo de referência e o Modelo 1.1 .

Figura 15: Ângulo de carga com Modelo de referência e Modelo 1.1.

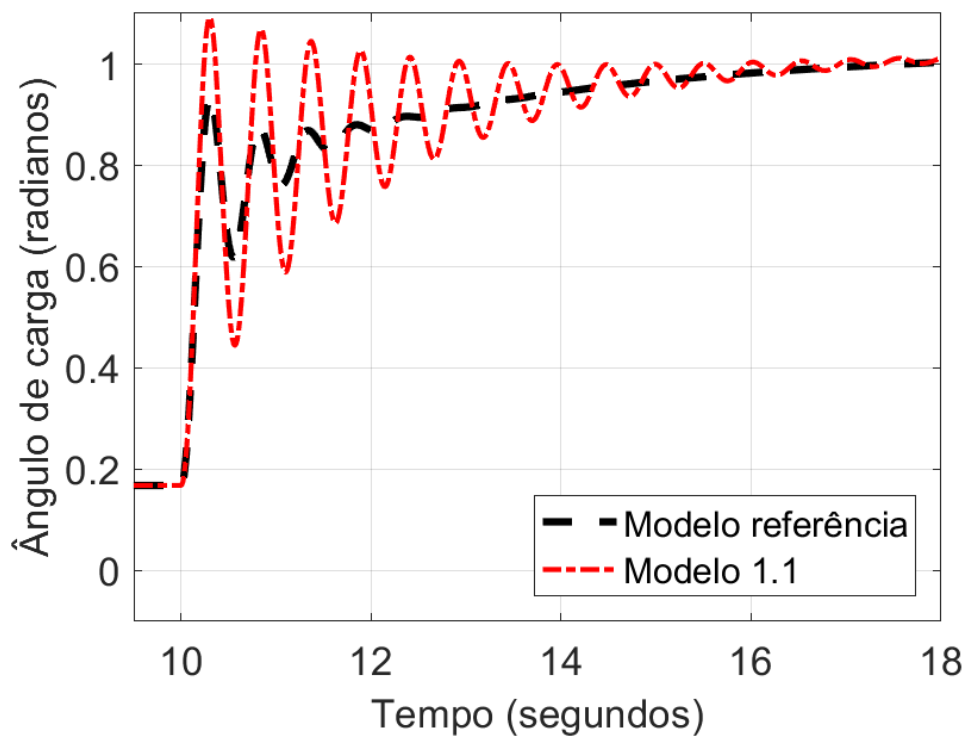




\section{Modelo 1.0}

Uma simplificação no Modelo 1.1 pode ser obtida ao reduzir uma bobina no eixo em quadratura do rotor fazendo $\mathrm{T}_{\mathrm{d}}=0, \bar{\psi}_{1 Q}=\bar{\psi}_{q}$, logo:

$$
\begin{aligned}
& \frac{d \bar{\psi}_{F}}{d_{t}}=\frac{1}{T_{d}^{\prime}}\left(\left(-\bar{\psi}_{F}+\bar{\psi}_{d}+\left(\frac{\bar{X}_{d}^{\prime}}{\bar{X}_{d}-\bar{X}_{d}^{\prime}}\right)\right) E_{f}\right) \\
& \frac{d \delta}{d_{t}}=\omega-\omega_{0} \\
& \frac{d \omega}{d_{t}}=\frac{\omega_{B}}{2 H}\left(T_{m}-\bar{\psi}_{d} \bar{i}_{q}+\bar{\psi}_{q} \bar{i}_{d}\right) \\
& \bar{i}_{d}=\frac{1}{\bar{X}_{d}{ }^{\prime \prime}} \bar{\psi}_{d}+\left(\frac{1}{\bar{X}_{d}}-\frac{1}{\bar{X}_{d}^{\prime}}\right) \bar{\psi}_{F} \\
& \bar{i}_{q}=\frac{1}{\bar{X}_{q}{ }^{\prime \prime}} \bar{\psi}_{q}
\end{aligned}
$$

Para simulação do Modelo 1.0 não há bobinas no rotor no eixo em quadratura, havendo apenas uma bobina no eixo direto, conforme ilustrado na Fig. 16.

Figura 16: Equação do fluxo no rotor no Modelo 1.0.

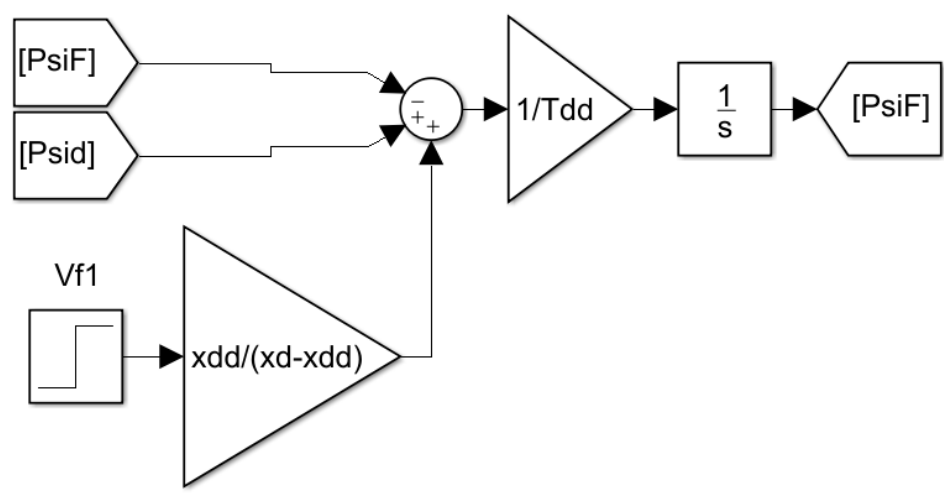


O diagrama das correntes no eixo direto e eixo em quadratura no Modelo 1.0 passa a ser conforme ilustrado na Fig. 17. Os fluxos magnéticos no estator permanecem conforme Fig. 7, tensões $\mathrm{v}_{\mathrm{d}}$ e $\mathrm{v}_{\mathrm{q}}$ conforme Fig. 3 e equações mecânicas conforme Fig. 4.

Figura 17: Comparativo entre o Modelo referência e Modelo 1.0.

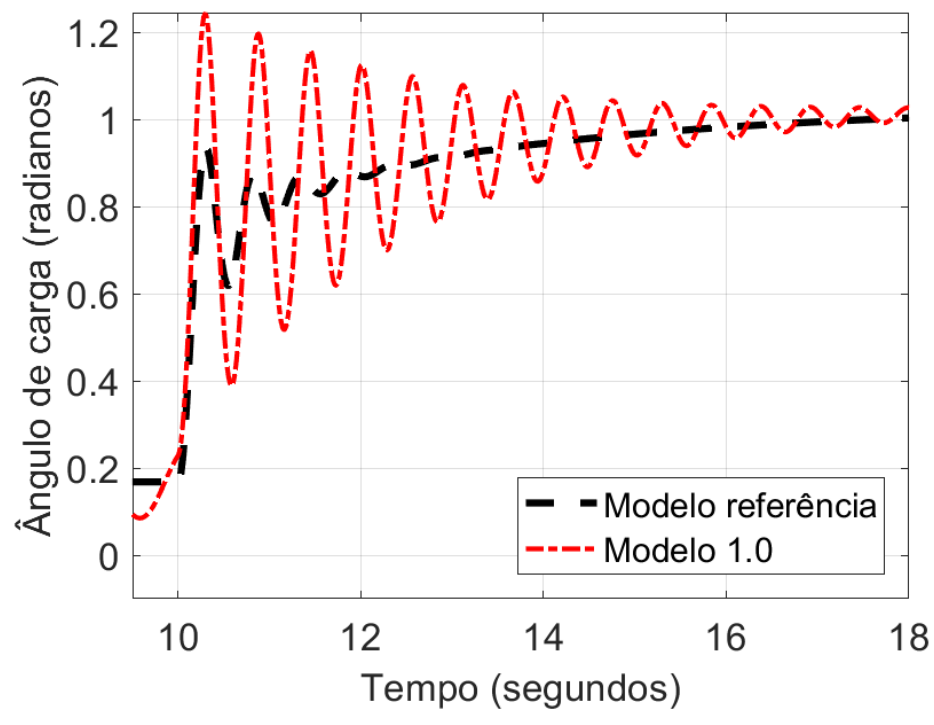

\section{Análise dos resultados obtidos com as simulações}

Durante a realização da simulação com o modelo de referência da Fig. 2, um bloco To workspace do SIMULINK é utilizado para produzir um vetor na área de trabalho do MATLAB. Na simulação com o modelo de referência da Fig. 2, esse vetor é salvo na área de trabalho do MATLAB com o nome "delta". Para a realização da simulação com o Modelo 2.2, um bloco To workspace do SIMULINK, ilustrado na Fig. 4, é utilizado para produzir um vetor na área de trabalho do MATLAB com o nome "delta2". Após realizada a simulação com os diferentes modelos matemáticos e construídos vetores que representam o comportamento do ângulo de carga é possível compará-los utilizando os coeficientes de correlação de Pearson. Esses coeficientes são utilizados para mensurar a correlação entre as variáveis apresentadas nos dois modelos. Os coeficientes de correlação de Pearson podem ter valores indo de -1 a +1 . Quando os coeficientes se aproximam de +1 indicam que há uma variação linear 
positiva entre as variáveis, ou seja, quando uma aumenta a outra também aumenta. Utiliza-se o comando corrcoef do MATLAB para comparar o vetor de referência aos vetores de cada um dos modelos. O intervalo de tempo dos vetores é determinado pelo passo de cálculo configurado no SIMULINK. Para as simulações aqui descritas utilizou-se o passo de cálculo de $1 \mathrm{E}^{-5} \mathrm{~s}$. Na Tabela 1 são apresentados os valores dos coeficientes de correlação de Pearson obtidos pela comparação dos diferentes modelos no intervalo de tempo de 10 a 25 segundos.

Tabela 1: Coeficientes de correlação de Pearson de 10 a 25 segundos.

\begin{tabular}{c|c}
\hline Parâmetro & Valor \\
\hline$\rho(\boldsymbol{\delta}$ Modelo Referência, $\boldsymbol{\delta}$ Modelo 2.2) & 0.9999 \\
$\rho(\boldsymbol{\delta}$ Modelo Referência, $\boldsymbol{\delta}$ Modelo 2.1) & 0.9576 \\
$\rho(\boldsymbol{\delta}$ Modelo Referência, $\boldsymbol{\delta}$ Modelo 1.1) & 0.9066 \\
$\rho(\boldsymbol{\delta}$ Modelo Referência, $\boldsymbol{\delta}$ Modelo 1.0) & 0.7441 \\
\hline
\end{tabular}

A Fig. 18 ilustra a comparação entre o ângulo de carga com os Modelos 2.2, 2.1, 1.1 e 1.0 no intervalo de tempo de 10 a 25 segundos. É possível observar que, em regime permanente, todos os modelos convergem para o mesmo resultado.

Figura 18: Comparativo entre Modelo de referência, Modelo 2.2, Modelo 2.1, Modelo 1.1 e Modelo 1.0 no intervalo de tempo de 10 a $25 \mathrm{~s}$.

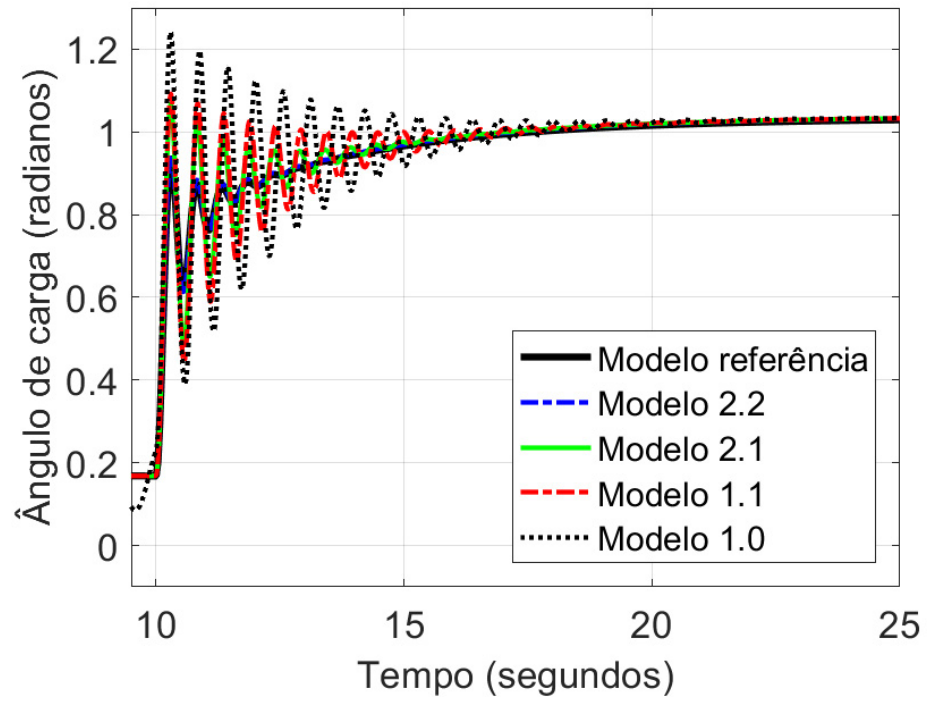


Na Tabela 2 são apresentados os valores dos coeficientes de Person obtidos pela comparação dos diferentes modelos no intervalo de tempo de 10 a 12 segundos. Utiliza-se novamente o comando corrcoef do MATLAB, considerando o novo intervalo de tempo, para comparar o vetor de referência aos vetores de cada um dos modelos.

Tabela 2: Coeficientes de correlação de Pearson de 10 a 12 segundos.

\begin{tabular}{c|c}
\hline Parâmetro & Valor \\
\hline$\rho(\boldsymbol{\delta}$ Modelo Referência, $\boldsymbol{\delta}$ Modelo 2.2) & 0.9998 \\
$\rho(\boldsymbol{\delta}$ Modelo Referência, $\boldsymbol{\delta}$ Modelo 2.1) & 0.9240 \\
$\rho(\boldsymbol{\delta}$ Modelo Referência, $\boldsymbol{\delta}$ Modelo 1.1) & 0.8658 \\
$\rho(\boldsymbol{\delta}$ Modelo Referência, $\boldsymbol{\delta}$ Modelo 1.0) & 0.6772 \\
\hline
\end{tabular}

A Fig. 19 ilustra a comparação entre o ângulo de carga obtido nas simulações e os Modelos 2.2, 2.1, 1.1 e 1.0 no intervalo de tempo de 10 a 12 segundos. É possível observar que, em regime transitório, há uma divergência entre os modelos.

Figura 19: Comparativo entre Modelo de referência, Modelo 2.2, Modelo 2.1, Modelo 1.1 e Modelo 1.0 no intervalo de tempo de 10 a $22 \mathrm{~s}$.

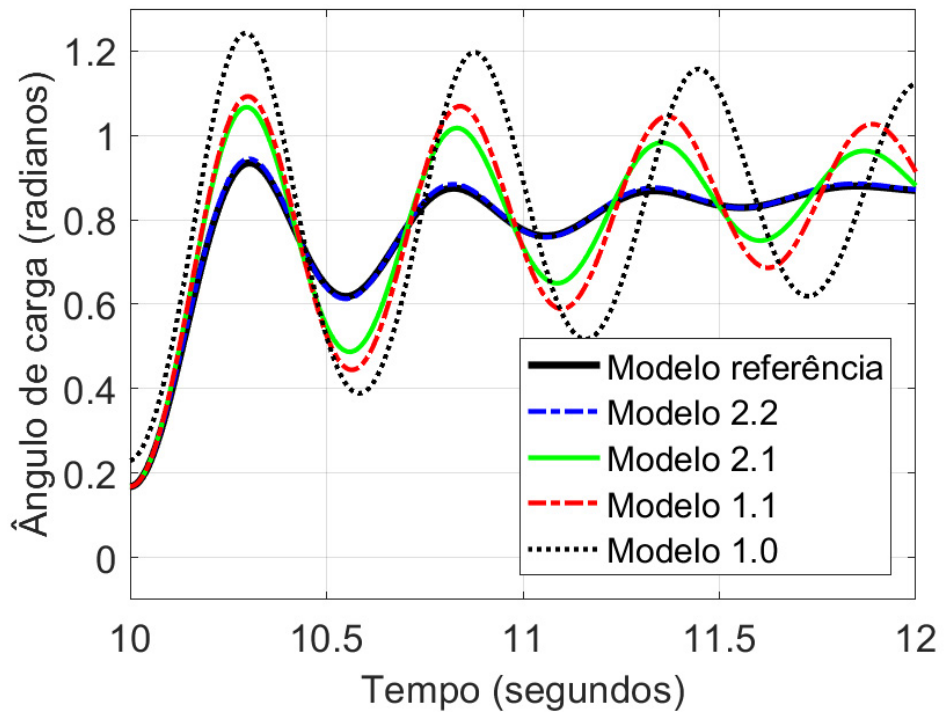

Logo, para identificação do ângulo de carga durante os momentos transitórios, é importante que os estudos sejam realizados considerando os modelos com maior número de bobinados do rotor, como o Modelo 2.2. A utilização de modelos simplificados 
com objetivo de facilitar o desenvolvimento de cálculos leva a um distanciamento dos parâmetros reais da máquina.

Em máquinas de polos salientes, o valor da reatância subtransitória do eixo em quadratura não é informado. Assim, para simulações de máquinas de polos salientes é preciso utilizar modelo 2.1 ou modelos simplificados, sendo que o modelo 2.2 utiliza-se apenas para máquinas de polos lisos.

É importante observar que nas máquinas de polos salientes serão informados os parâmetros $\mathrm{T}_{\mathrm{q}}$ e $\mathrm{T}_{\mathrm{q} \text { ", }}$ enquanto no modelo 2.1 são utilizados, nas simulações, os parâmetros $\mathrm{T}_{\mathrm{q}}$ e $\mathrm{T}_{\mathrm{q}}$. Diante disso, para simulações de máquinas de polos salientes, os valores fornecidos nas folhas de dados dos fabricantes para $\mathrm{T}_{\mathrm{q}^{\prime}}$ devem ser utilizados como $\mathrm{T}_{\mathrm{q}}$.

Após realizada uma análise dos resultados obtidos com os diferentes modelos, os alunos conseguiram observar que, à medida em que são consideradas simplificações no modelo matemático, os resultados para obtenção do ângulo de carga em regime transitório são comprometidos. Contudo, os resultados em regime permanente não são alterados. Nesse sentido, o modelo 1.0 pode ser compreendido como mais adequado para estudos em regime permanente, uma vez que fornece os mesmos resultados do modelo 2.2, ainda que com uma menor complexidade computacional. Já o modelo 2.2 é mais adequado para máquinas de polos lisos em estudos de regime transitório, e o modelo 2.1 é o mais adequado para máquinas de polos salientes.

\section{Conclusão}

Com a realização desta proposta de estudo, os alunos tiveram a oportunidade de visualizar, de forma objetiva, os principais modelos de máquinas síncronas utilizados na literatura, bem como o método para implementação desses modelos utilizando MATLAB/SIMULINK. Ademais, conseguiram observar as principais vantagens e desvantagens na utilização de modelos matemáticos simplificados. Não obstante, a realização da presente proposta com alunos da disciplina de máquinas elétricas possibilitou a aplicação interdisciplinar dos conhecimentos adquiridos no curso Técnico Subsequente em Eletromecânica. 


\section{A math models implementation of synchronous machines in MATLAB/SIMULINK as an interdisciplinary activity}

\section{Abstract}

In the discipline of electrical machines, offered in technical courses and engineering courses focused on the electrical area, one of the main components used in electrical power systems generation is studied, the synchronous machine. Understanding how this machine works requires prior knowledge of subjects such as physics and mathematics. Physics for the study of the moment of inertia, friction coefficient, interaction between magnetic fields, and mathematics for understanding the equations used in the construction of the models. Therefore, this topic, in addition to being very importance for understanding electrical systems, involves the application of knowledge acquired in several disciplines of the respective courses. For the realization of classes, some activities can be developed in the laboratory, however, there are some parameters on these machines that are inaccessible through practical experimentation and need to be analyzed via computer simulation. Among the possible software to perform these simulations is MATLAB I SIMULINK. In a proposal made with students of the Subsequent Technical course in Electromechanics, the present work was developed, which gathers the main mathematical models of synchronous machines and their implementation is described in MATLAB / SIMULINK. The proposed activity allowed students to better understand the principle of these machines operation.

Keywords: Load angle; Power system dynamics; Synchronous machine models; Power system models; Power system monitoring;.

\section{Referências}

BOLDEA, I. Synchronous Generators. Boca Raton: CRC Press, 2016.

GHAHREMANI, E. I. K. Online state estimation of a synchronous generator using unscented kalman filter from phasor measurements units. IEEE Transactions on Energy Conversion, v. 26 , n. 4, p. 1099-1108, 2011.

KRISHNA, S. An Introduction to Modelling of Power System Components. 1. ed. India: Springer, 2014.

JHA, S. M.; CHAKRABARTI, E. K. Estimation of the rotor angle of a synchronous generator by using PMU measurements. IEEE Eindhoven PowerTech, p. 1-6, 2015.

KUNDUR, P. Power System Stability and Control. New York: McGraw-Hill, 1994. (EPRI Power System Engineering Series).

VENKATASUBRAMANIAN, V. R. G. K. (2004). Direct computation of generator internal dynamic states from terminal measurements. In: 37TH ANNUAL HAWAII INTERNATIONAL CONFERENCE ON SYSTEM SCIENCES, Big Island, HI, USA, Anais [...], p. 6, 2004. 


\section{Anexo A - Valores dos parâmetros utilizados na simulação}

A Tabela 3 contém os parâmetros e unidades de medidas utilizados para simulações.

Tabela 3: Parâmetros utilizados nas simulações.

\begin{tabular}{c|c|c}
\hline Parâmetro & Valor & Dado em: \\
\hline $\mathrm{R}_{\mathrm{a}}$ & 0.003 & p.u. \\
$\mathrm{X}_{\mathrm{d}}$ & 1.8 & p.u. \\
$\mathrm{X}_{\mathrm{d}}^{\prime}$ & 0.3 & p.u. \\
$\mathrm{X}_{\mathrm{d}}^{\prime}$ & 0.23 & p.u. \\
$\mathrm{X}_{\mathrm{q}}$ & 1.7 & p.u. \\
$\mathrm{X}_{\mathrm{q}}^{\prime}$ & 0.65 & p.u. \\
$\mathrm{X}_{\mathrm{q}}^{\prime}$ & 0.25 & p.u. \\
$\mathrm{T}_{\mathrm{d}}^{\prime}$ & 0.8274 & segundos \\
$\mathrm{T}_{\mathrm{d}}^{\prime}$ & 0.0232 & segundos \\
$\mathrm{T}_{\mathrm{q}}$, & 0.3510 & segundos \\
$\mathrm{T}_{\mathrm{q}}^{\prime \prime}$ & 0.0293 & segundos \\
$\mathrm{H}$ & 3 & segundos \\
$\mathrm{V}$ & 1 & p.u. \\
\hline
\end{tabular}

